\title{
Proton beam therapy in non-small cell lung cancer: state of the art
}

\section{Hideyuki Harada \\ Shigeyuki Murayama}

Radiation and Proton Therapy Center, Shizuoka Cancer Center Hospital,

Nagaizumi, Shizuoka, Japan
This article was published in the following Dove Press journal:

Lung Cancer:Targets and Therapy

23 August 2017

Number of times this article has been viewed
Correspondence: Hideyuki Harada Shimonagakubo 1007, Nagaizumi-cho, Sunto-gun, Shizuoka 4I I-8777, Japan

Tel +81559895222

Fax +8I 559895783

Email h.harada@scchr.jp
Abstract: This review summarizes the past and present status of proton beam therapy (PBT) for lung cancer. PBT has a unique characteristic called the Bragg peak that enables a reduction in the dose of normal tissue around the tumor, but is sensitive to the uncertainties of density changes. The heterogeneity in electron density for thoracic lesions, such as those in the lung and mediastinum, and tumor movement according to respiration necessitates respiratory management for PBT to be applied in lung cancer patients. There are two types of PBT - a passively scattered approach and a scanning approach. Typically, a passively scattered approach is more robust for respiratory movement and a scanning approach could result in a more conformal dose distribution even when the tumor shape is complex. Large tumors of centrally located lung cancer may be more suitably irradiated than with intensity-modulated radiotherapy (IMRT) or stereotactic body radiotherapy (SBRT). For a locally advanced lung cancer, PBT can spare the lung and heart more than photon IMRT. However, no randomized controlled trial has reported differences between PBT and IMRT or SBRT for early-stage and locally advanced lung cancers. Therefore, a well-designed controlled trial is warranted.

Keywords: proton beam therapy, non-small cell lung cancer, survival, SBRT, IMRT

\section{Introduction}

In this review, we summarize the past and present statuses of proton beam therapy (PBT) for lung cancer. The source of the proton is the hydrogen nucleus and the proton is accelerated by an accelerator. The proton stops at a specific depth, called the Bragg peak, which significantly reduces the exit dose and the normal tissue damage around the tumor volume. In lung cancer, these characteristics could be beneficial in patients with cardiovascular disease, poor pulmonary function, or a history of previous thoracic radiotherapy.

\section{Proton therapy techniques}

Proton beam therapy is a type of radiotherapy with a unique characteristic that the proton stops at a specific depth according to its energy. As a result, the surrounding normal tissue dose is significantly reduced when compared with the tumor dose. In proton therapy, the dose is usually prescribed in units of cobalt gray equivalent (CGE). Because the relative biological effectiveness (RBE) should be considered, CGE is the physical dose multiplied by RBE. The RBE of the proton beam is defined as 1.1 .

There are two types of PBT; one is a passively scattered approach and the other is a scanning approach that enables intensity-modulated proton therapy (IMRT). The passive scattering method is similar to a photon three-dimensional radiotherapy plan. In the 
scanning approach, the tumor is irradiated spot-by-spot with different energy proton beams. The passive scattering method is more robust for thoracic malignancies that move during treatment because of respiration, causing density changes. In contrast, the passive scattering method has fewer advantages for complicated target shapes, and a scanning approach offers more flexible and conformal dose distributions.

\section{Motion management}

Proton beam therapy is sensitive to anatomic and density changes due to respiratory motion because of the characteristics of the Bragg peak. ${ }^{1}$ Therefore, respiratory motion evaluation and motion reduction is mandatory for patients with lung cancer treated by PBT. To evaluate respiratory tumor movement, four-dimensional (4D) computed tomography (CT) is necessary. A 4D-CT enables the radiation oncologist to measure an accurate internal tumor volume and to deliver radiation within a certain interval in the breathing cycle. Several studies reported that PBT plans based on the maximum intensity projection of end inhale, middle exhale, and end exhale images are the most robust. ${ }^{2}$ Alternate solutions for respiratory tumor motion is the gating method in which protons can be delivered only in a specific respiratory cycle such as the exhale phase. Typically, the passively scattered approach is more robust to respiratory movement, and the interplay effect between the scanning beam and the motion of the target can result in unintended inhomogeneous dose distributions in the target. Therefore, respiratory management such as gated irradiation is mandatory for scanning beam proton therapy in patients with lung cancer. ${ }^{3,4}$

\section{Adaptive planning}

The anatomy of the thoracic region could change during the course of PBT. Because the Bragg peak is sensitive to electron density and dose distribution, it is easily affected. Especially for stage III non-small cell lung cancer (NSCLC), adaptive planning is highly recommended because the tumor volume is often large, the treatment duration is over 6 weeks, and concurrent chemotherapy can effectively reduce tumor volume. For instance, in some patients in the MD Anderson phase II trial, the investigators compromised on tumor coverage to spare the esophagus and spinal cord. ${ }^{5}$

\section{Early-stage NSCLC}

The Loma Linda University initiated early-stage NSCLC proton therapy. The dose has escalated over time from 50 Gy (RBE) in 10 fractions to 70 Gy (RBE) in 10 fractions. $^{6,7}$ Following an early report, several Japanese centers studied hypofractionated PBT and reported promising local control rates of over $80 \%$. Early reports adopted a more fractionated schedule such as $80 \mathrm{~Gy}$ (RBE) in 20 fractions. Latter reports show that a less fractionated schedule was also effective. ${ }^{8-10}$

In contrast, stereotactic body photon radiotherapy (SBRT) shows significantly promising survival and local control rates for stage I NSCLC. In SBRT, a less fractionated schedule is adopted such as that with three or four fractions, and these results are similar to those of PBT. ${ }^{11,12}$ Therefore, the question is what advantage does PBT offer over SBRT. Small dosimetric differences were recorded between PBT and SBRT, except in tumors with a relatively large planning target volume (PTV) or patients with multiple tumors. ${ }^{13-15}$ Although dosimetric studies reported that some situations or tumors benefit more from PBT than SBRT, the question remains whether these benefits can translate into clinical benefits or not. From a recently reported systematic review, the survival with PBT (5-year survival: 60\%) was significantly better than with SBRT (5-year survival: 41\%). However, there was no significant difference after adding the percentage of operable patients. ${ }^{16}$ Moreover, surgery is reported to be associated with a significantly better outcome than SBRT for operable healthy patients (3-year survival; 55\%-60\% vs 29\%-52\%). ${ }^{17-19}$ Future research should identify clinical subgroups that will benefit from proton SBRT. Thus, the physician and patient should decide an indication of PBT together based on comprehensive clinical assessment.

\section{Specific situation}

In small lesions located in the peripheral lung, modern photon SBRT techniques such as cone-beam CT imageguided RT or motion management based on 4D-CT-based planning can achieve more conformal irradiation than previously used photon SBRT. Techniques of photon SBRT have become more mature and robust for tumor motion. Similarly, intensity-modulated proton-radiotherapy (IMPT) can deliver more conformal irradiation than passive PBT. Passive PBT is inferior in conformity because there is usually a need to add a widening margin and smear the compensator to mitigate range and setup uncertainties. ${ }^{15}$ In contrast, tumor motion management is mandatory in IMPT, and some concerns persist regarding the robustness of IMPT for moving tumors. ${ }^{20}$ From a clinical standpoint, there is no difference between photon SBRT and PBT and, therefore, small peripheral tumors could be treated effectively by both photon SBRT and PBT. On the other hand, for larger tumors such as T2N0M0, normal lung doses around the tumor are increased in photon SBRT. However, Iwata et al reported that no patient developed 
grade 3 or higher pulmonary toxicities in 43 patients with T2 tumors treated by PBT. Therefore, large tumors are good candidates for PBT. ${ }^{21,22}$

In centrally located tumors within $2 \mathrm{~cm}$ from the proximal bronchial tree, photon SBRT studies have reported significantly high rates of grade 3 or higher toxicities. ${ }^{23}$ In the RTOG 0813 study, preliminary results showed that 4 of 81 patients died from side effects when a high SBRT dose was delivered. PBT is, therefore, advantageous for central tumors adjacent to the heart, esophagus, and bronchus. ${ }^{24}$ In a dosimetric study, PBT plans significantly reduced the mean total lung dose from 5.4 to $3.5 \mathrm{~Gy}(p<0.001)$ and $2.8 \mathrm{~Gy}(p$ $<0.001)$, and reduced the total lung volume receiving 5,10 , and $20 \mathrm{~Gy}(p<0.001)$ as compared to photon SBRT plans (50 Gy in four fractions). However, PBT could not reduce the major bronchi or pulmonary artery doses in cases of tumors adjacent to these organs. ${ }^{25}$

In clinical studies, Japanese centers reported promising local control and feasibility for central tumors using a more fractionated schedule PBT. ${ }^{26,27}$ Makita et $\mathrm{al}^{27}$ reported that they treated 24 central tumors with 80 Gy (RBE) in 25 fractions, and no one experienced late grade 3 or severe toxicities. These promising results have some limitations in that these are retrospective studies with a small number of patients. Therefore, a multi-institutional prospective cohort study or randomized controlled trial comparing PBT and IMRT or SBRT is necessary.

\section{Locally advanced NSCLC}

Current standard of care for locally advanced NSCLC is concurrent chemotherapy and thoracic radiotherapy. Thoracic radiotherapy has developed over the past three decades. Initially, three doses were compared in the RTOG 7301 trial (40, 50 , and $60 \mathrm{~Gy}$ ); 60 Gy showed the most promising survival and local control. ${ }^{28}$ Sequentially, the development of che- motherapy has been evaluated, and concurrent chemoradiation has shown the most promising survival as compared to sequential chemoradiation or thoracic radiation. ${ }^{29,30}$ Recently, the RTOG 0617 trial comparing 74 Gy versus 60 Gy photon radiotherapy concurrent with chemotherapy unfortunately showed shorter survival in the 74 Gy arm. The definitive reason has not been reported yet, but the combined toxicities (lung, heart, or esophagus) are believed to be the reason why the high dose failed.

From a dosimetric study, the PBT plan is better in terms of the radiation dose to organs at risk such as the heart and lungs. Figure 1 shows a demonstrative case comparing 3D conformal photon radiotherapy, IMRT, and PBT. PBT spares more of the lung and heart than three-dimensional conformal radiotherapy (3DCRT). ${ }^{31}$ Further, several clinical trials using PBT for locally advanced NSCLC showed promising survival even when prescribing higher doses than $60 \mathrm{~Gy} .{ }^{32,33}$ However, several groups reported severe esophageal toxicities when using high-dose proton therapy. Even for PBT, dose escalation for stage III NSCLC continues to be challenging. ${ }^{34,35}$

In contrast, IMRT has developed, and its clinical outcomes were more promising than 3DCRT in the RTOG 0617 trial. In particular, IMRT reduced the heart and lung doses and improved quality of life, compared with 3DCRT. ${ }^{36}$ Therefore, a well-designed randomized trial comparing IMRT and PBT is needed. Recently, the results of a Bayesian randomized trial comparing IMRT versus passively scattered PBT for locally advanced NSCLC were reported. They assessed rates of, and time to, treatment failure (TF) defined as either a grade $\geq 3$ radiation pneumonitis or local recurrence within 12 months. Unfortunately, they failed to demonstrate a difference between groups (TF rates at 12 months were $15.6 \%$ in IMRT group and $24.6 \%$ in the PBT group). ${ }^{37}$ This is a phase II trial and the primary endpoint is not survival; therefore, we are unable to come
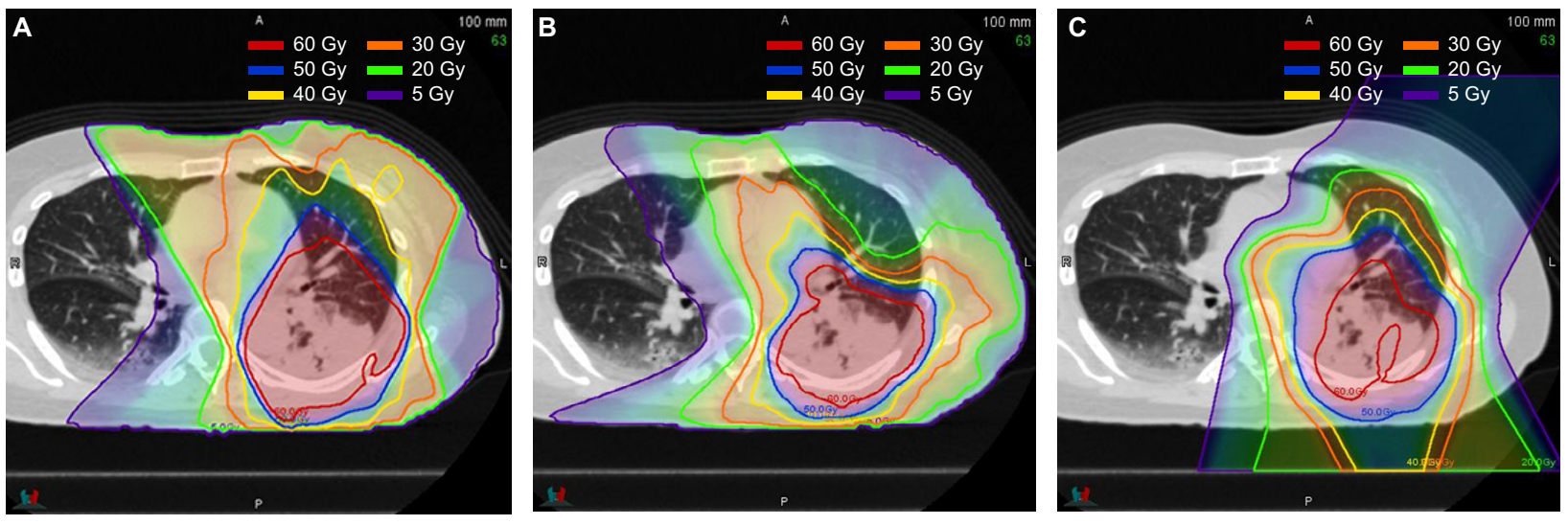

Figure I Demonstrative cases comparing (A) 3DCRT, (B) IMRT, and (C) PBT.

Note: Images courtesy of Tatsuya Segawa.

Abbreviations: 3DCRT, three-dimensional conformal radiotherapy; IMRT, intensity-modulated radiotherapy; PBT, proton beam therapy. 
to any conclusion at this time. The results of ongoing trials comparing IMRT and PBT should be analyzed before concluding if PBT is superior to IMRT.

\section{Re-irradiation}

As patients with lung cancer begin to live longer, recurrence in the previously irradiated area or adjacent area can occur. This is a challenging situation because of limitations of the normal tissue tolerance. In patients with NSCLC treated with concurrent chemoradiation to the thorax, 5-year rates of locoregional recurrence approached $30 \%{ }^{38}$ Higher re-irradiation doses could be considered, but the results of photon re-irradiation remain unsatisfactory. ${ }^{39-41}$ Modern radiotherapy techniques such as IMRT or PBT report promising results for re-irradiation for locoregionally recurrent lung cancer. Although further evaluation is needed, PBT is beneficial, especially for mediastinal lymph nodes. ${ }^{42}$

\section{Conclusion}

The unique characteristics of the Bragg peak are advantageous in RT for lung cancer because it is compatible with a sufficient tumor dose to improve local control and survival while sparing normal organs at risk, such as the heart, lung, and spinal cord. In addition to the passive scatter PBT technique, IMPT is now available. However, the clinical benefit of PBT over photon-based modern RT techniques such as SBRT or IMRT has never been established before and, therefore, a well-designed clinical trial is warranted for further study.

\section{Disclosure}

The authors report no conflicts of interest in this work.

\section{References}

1. Zhao L, Sandison GA, Farr JB, Hsi WC, Li XA. Dosimetric impact of intrafraction motion for compensator-based proton therapy for lung cancer. Phys Med Biol. 2008;53(12):3343-3364.

2. Wang N, Patyal B, Ghebremedhin A, Bush D. Evaluation and comparison of new 4DCT based strategies for proton treatment planning for lung tumors. Radiat Oncol. 2013;8:73.

3. Bert C, Gemmel A, Saito N, Rietzel E. Gated irradiation with scanned particle beams. Int J Radiat Oncol Biol Phys. 2009;73(4):1270-1275.

4. Kanehira T, Matsuura T, Takao S, et al. Impact of real-time image gating on spot scanning proton therapy for lung tumors: a simulation study. Int J Radiat Oncol Biol Phys. 2017;97(1):173-181.

5. Koay EJ, Lege D, Mohan R, Komaki R, Cox JD, Chang JY. Adaptive/ nonadaptive proton radiation planning and outcomes in a phase II trial for locally advanced non-small cell lung cancer. Int J Radiat Oncol Biol Phys. 2012;84(5):1093-1100.

6. Bush DA, Slater JD, Bonnet R, et al. Proton-beam radiotherapy for early-stage lung cancer. Chest. 1999;116(5):1313-1319.

7. Bush DA, Cheek G, Zaheer S, et al. High-dose hypofractionated proton beam radiation therapy is safe and effective for central and peripheral early-stage non-small cell lung cancer: results of a 12-year experience at Loma Linda University Medical Center. Int J Radiat Oncol Biol Phys. 2013;86(5):964-968.
8. Shioyama Y, Tokuuye K, Okumura T, et al. Clinical evaluation of proton radiotherapy for non-small-cell lung cancer. Int J Radiat Oncol Biol Phys. 2003;56(1):7-13.

9. Nihei K, Ogino T, Ishikura S, Nishimura H. High-dose proton beam therapy for Stage I non-small-cell lung cancer. Int J Radiat Oncol Biol Phys. 2006;65(1):107-111.

10. Nakayama $H$, Sugahara S, Tokita M, et al. Proton beam therapy for patients with medically inoperable stage I non-small-cell lung cancer at the University of Tsukuba. Int J Radiat Oncol Biol Phys. 2010;78(2):467-471.

11. Timmerman R, Paulus R, Galvin J et al. Stereotactic body radiation therapy for inoperable early stage lung cancer. JAMA. 2010;303(11):1070-1076.

12. Nagata $Y$, Hiraoka M, Shibata T, et al. Prospective trial of stereotactic body radiation therapy for both operable and inoperable T1N0M0 non-small cell lung cancer: Japan Clinical Oncology Group Study JCOG0403. Int J Radiat Oncol Biol Phys. 2015;93(5):989-996.

13. Georg D, Hillbrand M, Stock M, Dieckmann K, Pötter R. Can protons improve SBRT for lung lesions? Dosimetric considerations. Radiother Oncol. 2008;88(3):368-375.

14. Kadoya N, Obata Y, Kato T, et al. Dose-volume comparison of proton radiotherapy and stereotactic body radiotherapy for non-small-cell lung cancer. Int J Radiat Oncol Biol Phys. 2011;79(4):1225-1231.

15. Seco J, Panahandeh HR, Westover K, Adams J, Willers H. Treatment of non-small cell lung cancer patients with photon beam-based stereotactic body radiotherapy: dosimetric comparison with photon plans highlights importance of range uncertainty. Int J Radiat Oncol Biol Phys. 2012;83(1):354-361.

16. Chi A, Chen H, Wen S, Yan H, Liao Z. Comparison of particle beam therapy and stereotactic body radiotherapy for early stage non-small cell lung cancer: a systematic review and hypothesis-generating metaanalysis. Radiother Oncol. 2017;123(3):346-354

17. Crabtree TD, Puri V, Robinson C, et al. Analysis of first recurrence and survival in patients with stage I non-small cell lung cancer treated with surgical resection or stereotactic radiation therapy. JThorac Cardiovasc Surg. 2014;147(4):1183-1191; discussion 1191-1192.

18. Matsuo Y, Chen F, Hamaji M, et al. Comparison of long-term survival outcomes between stereotactic body radiotherapy and sublobar resection for stage I non-small-cell lung cancer in patients at high risk for lobectomy: a propensity score matching analysis. Eur J Cancer. 2014;50(17): 2932-2938.

19. Rosen JE, Salazar MC, Wang Z, et al. Lobectomy versus stereotactic body radiotherapy in healthy patients with stage I lung cancer. JThorac Cardiovasc Surg. 2016;152(1):44-54.e9.

20. Albertini F, Bolsi A, Lomax AJ, Rutz HP, Timmerman B, Goitein G. Sensitivity of intensity modulated proton therapy plans to changes in patient weight. Radiother Oncol. 2008;86(2):187-194.

21. Koto M, Takai Y, Ogawa Y, et al. A phase II study on stereotactic body radiotherapy for stage I non-small cell lung cancer. Radiother Oncol. 2007;85(3):429-434.

22. Iwata H, Demizu Y, Fujii O, et al. Long term outcome of proton therapy and carbon-ion therapy for large (T2a-T2bN0M0) non-small cell lung cancer. J Thorac Oncol. 2013;8(6):726-735.

23. Timmerman R, McGarry R, Yiannoutsos C, et al. Excessive toxicity when treating central tumors in a phase II study of stereotactic body radiation therapy for medically inoperable early-stage lung cancer. $J$ Clin Oncol. 2006;24(30):4833-4839.

24. Bezjak A, Paulus R, Gaspar LE, et al. Primary study endpoint analysis for NRG Oncology/RTOG 0813 trial of stereotactic body radiation therapy (SBRT) for centrally located non-small cell lung cancer (NSCLC). Int J Radiat Oncol Biol Phys. 2016;94(1):5-6.

25. Register SP, Zhang X, Mohan R, Chang JY. Proton stereotactic body radiation therapy for clinically challenging cases of centrally and superiorly located stage I non-small-cell lung cancer. Int J Radiat Oncol Biol Phys. 2011;80(4):1015-1022.

26. Kanemoto A, Okumura T, Ishikawa H, et al. Outcomes and prognostic factors for recurrence after high-dose proton beam therapy for centrally and peripherally located stage I non-small-cell lung cancer. Clin Lung Cancer. 2014;15(2):e7-e12. 
27. Makita C, Nakamura T, Takada A, et al. High-dose proton beam therapy for stage I non-small cell lung cancer: clinical outcomes and prognostic factors. Acta Oncol. 2015;54(3):307-314.

28. Perez CA, Stanley K, Rubin P, et al. A prospective randomized study of various irradiation doses and fractionation schedules in the treatment of inoperable non-oat-cell carcinoma of the lung. Preliminary report by the Radiation Therapy Oncology Group. Cancer. 1980;45(11):2744-2753.

29. Furuse K, Fukuoka M, Kawahara M, et al. Phase III study of concurrent versus sequential thoracic radiotherapy in combination with mitomycin, vindesine, and cisplatin in unresectable stage III non-small-cell lung cancer. J Clin Oncol. 1999;17(9):2692-2699.

30. Curran WJ Jr, Paulus R, Langer CJ, et al. Sequential vs. concurrent chemoradiation for stage III non-small cell lung cancer: randomized phase III trial RTOG 9410. J Natl Cancer Inst. 2011;103(19):1452-1460.

31. Ohno T, Oshiro Y, Mizumoto M, et al. Comparison of dose-volume histograms between proton beam and $\mathrm{X}$-ray conformal radiotherapy for locally advanced non-small-cell lung cancer. J Radiat Res. 2015;56(1): $128-133$.

32. Oshiro Y, Okumura T, Kurishima K, et al. High-dose concurrent chemoproton therapy for Stage III NSCLC: preliminary results of a Phase II study. J Radiat Res. 2014;55(5):959-965.

33. Chang JY, Komaki R, Lu C, et al. Phase 2 study of high-dose proton therapy with concurrent chemotherapy for unresectable stage III nonsmall cell lung cancer. Cancer. 2011;117(20):4707-4713.

34. Gomez DR, Gillin M, Liao Z, et al. Phase 1 study of dose escalation in hypofractionated proton beam therapy for non-small cell lung cancer. Int J Radiat Oncol Biol Phys. 2013;86(4):665-670.
35. Harada H, Fuji H, Ono A, et al. Dose escalation study of proton beam therapy with concurrent chemotherapy for stage III non-small cell lung cancer. Cancer Sci. 2016;107(7):1018-1021.

36. Chun SG, Hu C, Choy $\mathrm{H}$, et al. Outcome of intensity modulated and 3D-conformal radiotherapy for stage III non-small cell lung cancer in NRG oncology/RTOG0617. Poster Presented at: 16th World Conference on Lung Cancer; September 6-10; 2015; Denver, CO.

37. Liao ZX, Lee JJ, Komaki R, et al. Bayesian randomized trial comparing intensity modulated radiation therapy versus passively scattered proton therapy for locally advanced non-small cell lung cancer. J Clin Oncol. 2016;34(Suppl 15): abstr 8500.

38. Aupérin A, Le Péchoux C, Rolland E, et al. Meta-analysis of concomitant versus sequential radiochemotherapy in locally advanced non-small-cell lung cancer. J Clin Oncol. 2010;28(13):2181-2190.

39. Jeremić B, Videtic GM. Chest reirradiation with external beam radiotherapy for locally recurrent non-small-cell lung cancer: a review. Int J Radiat Oncol Biol Phys. 2011;80(4):969-977.

40. De Ruysscher D, Faivre-Finn C, Le Pechoux C, Peeters S, Belderbos J. High-dose re-irradiation following radical radiotherapy for non-smallcell lung cancer. Lancet Oncol. 2014;15(13):e620-e624.

41. Sumita K, Harada H, Asakura H, et al. Re-irradiation for locoregionally recurrent tumors of the thorax: a single-institution, retrospective study. Radiat Oncol. 2016;11:104.

42. McAvoy S, Ciura K, Wei C, et al. Definitive reirradiation for locoregionally recurrent non-small cell lung cancer with proton beam therapy or intensity modulated radiation therapy: predictors of high-grade toxicity and survival outcomes. Int J Radiat Oncol Biol Phys. 2014;90(4):819-827.
Lung Cancer: Targets and Therapy

\section{Publish your work in this journal}

Lung Cancer: Targets and Therapy is an international, peer-reviewed, open acces journal focusing on lung cancer research, identification of therapeutic targets and the optimal use of preventative and integrated treatment interventions to achieve improved outcomes, enhanced survival and quality of life for the cancer patient. Specific topics covered in the journal include: Epidemiology, detection and screening Cellular research and biomarkers; Identification of biotargets and agents with novel

Submit your manuscript here: https://www.dovepress.com/lung-cancer-targets--therapy-journa
Dovepress

mechanisms of action; Optimal clinical use of existing anticancer agents, including combination therapies; Radiation and surgery; Palliative care; Patient adherence, quality of life, satisfaction; Health economic evaluations. The manuscript management system is completely online and includes a very quick and fair peer-review system. Visit http://www.dovepress.com/testimonials.php to read real quotes from published authors. 